\title{
Fasting Glucagon Level in Type 2 Diabetes and Impaired Glucose Tolerance and Its Association With Diabetes-Associated Clinical Parameters: A Study From Karachi, Pakistan
}

Eraj Abbas ${ }^{1}$, Iftikhar Ahmed Siddiqui ${ }^{1}$, Muhammad Saeed Khan ${ }^{1}$, Kahkashan Perveen ${ }^{1}$, Anum Butt ${ }^{2}$, Asher Fawwad $^{2}$

1. Biochemistry, Baqai Medical University, Karachi, PAK 2. Research, Baqai Institute of Diabetology and Endocrinology, Baqai Medical University, Karachi, PAK

Corresponding author: Asher Fawwad, asherfawwad@bide.edu.pk

\section{Abstract}

\section{Aim and objective}

The study aims to analyze fasting glucagon in patients with type 2 diabetes and impaired glucose tolerance and correlate it with anthropometric and biochemical parameters in a large proportion of Pakistani people with diabetes.

\section{Methodology}

The participants of the study were categorized into three groups based on oral glucose tolerance test, as per American Diabetes Association guidelines. Group A consisted of normal glucose tolerance subjects $(n=30)$, Group B consisted of subjects with impaired glucose tolerance $(n=30)$, and Group $C$ had full-blown subjects with type 2 diabetes $(n=30)$. Biochemical parameters, such as fasting glucagon, fasting plasma and 2-hour glucose, glycated hemoglobin, and lipid profile, and anthropometric parameters, such as body mass index (BMI), waist and hip circumference, waist-to-hip ratio, and systolic and diastolic blood pressure, were measured.

\section{Results}

The mean values of fasting glucagon level in Group A, Group B, and Group C were 39.24 $\pm 4.5,44.5 \pm 8.25$, and $49.02 \pm 9.15 \mathrm{pg} / \mathrm{ml}$, respectively. Statistically significant difference was not found in fasting glucagon level among these groups (p-value 0.614 ). Fasting glucagon was positively and independently correlated with 2hour plasma glucose, systolic blood pressure, diastolic blood pressure, BMI, hip and waist circumference, and hip-to-waist ratio in Group C. In Group B, fasting glucagon was positively correlated with 2-hour plasma glucose, BMI, and hip circumference, while it was not correlated with fasting plasma glucose in both groups. In Group A, fasting glucagon found positively correlated with systolic blood pressure and hip circumference.

Review began 09/10/2020 Review ended 02/04/2021 Published 02/18/2021

\section{() Copyright 2021}

Abbas et al. This is an open access article distributed under the terms of the Creative Commons Attribution License CC-BY 4.0., which permits unrestricted use, distribution, and reproduction in any medium, provided the original author and source are credited.

\section{Conclusion}

Our observation suggests that fasting plasma glucose is not concomitant with glucagon levels; however, glucagon suppression, after glucose intake, was dysregulated in type 2 diabetes and impaired glucose tolerance. Moreover, glucagon is associated with central obesity in type 2 diabetic patients.

Categories: Endocrinology/Diabetes/Metabolism, Public Health, Other Keywords: glucagon, type 2 diabetes, impaired glucose tolerance, obesity

\section{Introduction}

The primary cause of type 2 diabetes mellitus (T2DM) is insulin resistance and relative insulin deficiency; however, many subjects also exhibit inappropriate levels of circulating glucagon [1]. Important evidence for this was the demonstration that subjects with T2DM had higher baseline glucagon levels than subjects without diabetes, and these levels were not suppressed by a carbohydrate meal, as was the case in subjects without diabetes [2].

Glucagon elevates the concentration of glucose in the blood upon fasting by activating gluconeogenesis (i.e., the hepatic production of glucose through a non-carbohydrate source) and by the breakdown of glycogen [3]. Regulation of glucagon secretion is impaired in subjects with T2DM who have elevated plasma glucagon concentrations in the fasting state and defective postprandial glucagon suppression that results in undesirably high plasma glucagon concentrations in the context of hyperglycemia [4]. Lack of suppression of 
postprandial glucagon secretion in subjects with T2DM plays an important role in the pathogenesis of postprandial hyperglycemia [5].

Thus, hyperglucagonemia contributes to the increased hepatic glucose production that distinguishes subjects with T2DM. Furthermore, in subjects with T2DM, plasma glucagon concentrations may even increase in response to a meal [6]. The mechanisms accountable for increased glucagon levels under hyperglycemic conditions in subjects with T2DM may include impaired glucose sensing by alpha cells and/or resistance of pancreatic alpha cells to the inhibitory actions of insulin [7].

Obesity is associated with insulin resistance and increases the risk of T2DM development. An excess of adipose tissue induces insulin resistance and pancreatic beta-cell dysfunction modulated by releasing nonesterified fatty acids; endocrine hormones, such as leptin and adipokine; and proinflammatory cytokines [8]. Stern et al. [9] recently reported that the dysregulation of glucagon secretion in obesity leads to glucoregulatory disturbance in mice. In contrast, glucagon appears to promote satiety and increased energy expenditure in obese individuals, which may contribute to weight loss. Also, insulin and glucagon have a role in energy balance regulation [10,11]. The current study is, therefore, designed to analyze serum glucagon levels in subjects with T2DM and impaired glucose tolerance compared to normal glucose tolerance and to evaluate the correlation of fasting glucagon with fasting and 2-hour plasma glucose (2$\mathrm{hPG}$ ), glycated hemoglobin (HbA1c), lipid profile, and anthropometric parameters specifically with the parameters of central obesity in the study population.

\section{Materials And Methods}

The study was conducted at Baqai Institute of Diabetology and Endocrinology, Baqai Medical University (BMU), Karachi for the duration of January 2016 to January 2018. Convenience sampling for the research purpose was used from the available pool of respondents. The participants of this study were categorized into three groups on the basis of oral glucose tolerance test (OGTT), as per American Diabetes Association (ADA) guidelines. Group A consisted of normal glucose tolerance subjects $(n=30)$, Group B consisted of subjects with impaired glucose tolerance $(n=30)$, and Group $C$ had full-blown subjects with T2DM $(\mathrm{n}=30)$. Normal glucose tolerance was taken as fasting plasma glucose level (FPG) <100 mg/dl and 2-hPG level $<140 \mathrm{mg} / \mathrm{dl}$. Impaired glucose tolerance was considered as FPG level 100-125 mg/dl and 2-hPG level $140-199 \mathrm{mg} / \mathrm{dl}$. Diabetes was considered as FPG level $\geqslant 126 \mathrm{mg} / \mathrm{dl}$ and 2-hPG level $\geqslant 200 \mathrm{mg} / \mathrm{dl}$ as per ADA guidelines [12]. Subjects with known comorbidities such as cancer or other debilitating disorders such as cardiopulmonary disorders, an autoimmune disorder, type 1 diabetes, and gestational diabetes were excluded. The study was carried out after ethical approval from the ethics committee of BMU.

\section{Anthropometric measures}

Weight, height, waist and hip circumference, and blood pressure were measured using standardized methods [13]. Body mass index (BMI) was calculated as weight in kilograms divided by height in meters squared $\left(\mathrm{kg} / \mathrm{m}^{2}\right)$. Waist circumference was measured between the center point of the lower margin of the ribs and the mid-point of the iliac crest. Hip circumference was measured around the widest portion of the buttocks, with the tape parallel to the floor [14]. Blood pressure was measured using an auscultatory method with the help of a stethoscope and a sphygmomanometer [15].

\section{Clinical measures}

Biochemical parameters such as fasting and 2-hPG, HbA1c, lipid profile, and glucagon were assessed by standardized methods. Fasting glucagon was estimated by the glucagon enzyme immunoassay using human glucagon enzyme-linked immunosorbent assay kit (Genorise Scientific, Inc., PA, USA) [2]. Fasting plasma glucose and 2-hPG levels were measured using glucose oxidase peroxidase [16]; total cholesterol was estimated using the cholesterol oxidase phenol 4-amino antipyrine peroxidase method, and triglycerides were measured using the glycerol phosphate oxidase-p-aminophenazone method. High-density lipoprotein cholesterol (HDL-C) was estimated using the Immuno FS method, low-density lipoprotein cholesterol (LDLC) was measured by the direct method [17], and HbA1c was measured by high-performance liquid chromatography [18].

\section{Statistical analysis}

All statistical analyses were performed using IBM SPSS Statistics for Windows, Version 20.0. (IBM Corp., Armonk, NY, USA). The anthropometric and clinical variables were analyzed by using either the one-way analysis of variance or Student's t-test, Mann-Whitney U test or Kruskal-Wallis H test, and chi-square test as appropriate. The degree of correlation between metabolic parameters and glucagon was presented by the Pearson correlation coefficient. Multiple linear regression analyses assessed the independent predictors of glucagon. Results were considered statistically significant at a p-value $<0.05$.

\section{Results}

The mean of anthropometric and biochemical parameters among study groups is compared in Table 1 . It is shown that mean age, weight, hip and waist circumference, and systolic blood pressure were significantly 


\section{Cureus}

higher in Group C subjects as compared to Group A, whereas no significant difference was found in any parameter between Group B and Group C. The mean difference of fasting glucagon was not found significant among the study group. Fasting plasma glucose and 2-hPG levels were significantly higher in Group C and Group B ( $p$-value < 0.0001 ) as compared to Group A. Cholesterol, triglycerides, and LDL level were significantly higher in Group C as compared to Group A, while HDL level was lower, respectively. HbA1c was found significantly higher in Group $\mathrm{C}$ as compared to the other two groups.

\begin{tabular}{|c|c|c|c|}
\hline Parameters & Group A & Group B & Group C \\
\hline Age (years) & $44.17 \pm 12.86$ & $45.79 \pm 12.6$ & $52.03 \pm 9.88^{\star \star}$ \\
\hline BMI $\left(\mathrm{kg} / \mathrm{m}^{2}\right)$ & $26.21 \pm 4.53$ & $28 \pm 5.51$ & $27.8 \pm 6.4$ \\
\hline Waist circumference $(\mathrm{cm})$ & $91.48 \pm 12.79$ & $92.7 \pm 11.84$ & $98.67 \pm 14.35^{\star \star}$ \\
\hline Hip circumference $(\mathrm{cm})$ & $67.76 \pm 14.42$ & $102.73 \pm 18.21$ & $105.63 \pm 12.9^{\star \star}$ \\
\hline SBP (mmHg) & $120.9 \pm 17.02$ & $125.17 \pm 17.65$ & $131.87 \pm 23.01^{\star \star}$ \\
\hline $\mathrm{DBP}(\mathrm{mmHg})$ & $81.17 \pm 10.69$ & $81.03 \pm 9.39$ & $85.53 \pm 10.89$ \\
\hline Fasting glucagon (pg/ml) & $39.24 \pm 4.5$ & $44.51 \pm 8.25$ & $49.02 \pm 9.15$ \\
\hline Fasting plasma glucose (mg/dl) & $87.83 \pm 10.44$ & $107.73 \pm 10.85^{\star}$ & $202.97 \pm 10.23^{\star \star}$ \\
\hline Two-hour plasma glucose (mg/dl) & $100.93 \pm 21.46$ & $145.97 \pm 16.89^{\star}$ & $275.64 \pm 13.32^{\star *}$ \\
\hline Cholesterol (mg/dl) & $220.43 \pm 14.17$ & $236.43 \pm 12.31$ & $248.9 \pm 9.74^{\star \star}$ \\
\hline Triglyceride (mg/dl) & $163.63 \pm 9.21$ & $203.1 \pm 8.49^{\star}$ & $216.37 \pm 14.21^{\star \star}$ \\
\hline $\mathrm{HDL}(\mathrm{mg} / \mathrm{dl})$ & $38.43 \pm 10.49$ & $36.97 \pm 5.93$ & $20.47 \pm 6.28^{\star \star}$ \\
\hline LDL (mg/dl) & $115.53 \pm 12.51$ & $108.83 \pm 6.19$ & $111.07 \pm 12.31^{\star \star}$ \\
\hline HbA1c (mg/dl) & $5.35 \pm 0.56$ & $5.52 \pm 0.7 \dagger$ & $6.83 \pm 1.82$ \\
\hline
\end{tabular}

TABLE 1: Comparison of the baseline anthropometric and biochemical characteristics between Group A, Group B, and Group C

$p$-Value $\leq 0.05$ is taken as significant; ${ }^{\star} p \leq 0.05$ (Group A vs. Group B); ${ }^{\star \star} p \leq 0.05$ (Group A vs. Group C); ${ }^{*} p \leq 0.05 ;$ † $\leq \leq .05$ (Group B vs. Group C).

BMI, body mass index; SBP, systolic blood pressure; DBP, diastolic blood pressure; HDL, high-density lipoprotein; LDL, low-density lipoprotein; HbA1c, glycated hemoglobin.

Group A: Patients with normal glucose tolerance.

Group B: Patients with impaired glucose tolerance.

Group C: Patients with type 2 diabetes.

The statistics of correlation were applied to investigate the effect of fasting glucagon on anthropometrics and biochemical parameters in study groups. The incidence of positive correlation was found for fasting glucagon with 2-hPG, systolic blood pressure, diastolic blood pressure, BMI, waist circumference, and waistto-hip ratio in Group C ( $\mathrm{p}<0.05)$. The fasting glucagon was positively correlated with 2-hPG and BMI in Group B and with systolic blood pressure in Group A. However, fasting glucagon was positively associated with hip circumference among all groups $(\mathrm{p}<0.05)$ (Table 2). 


\section{Cureus}

\begin{tabular}{|c|c|c|c|c|c|c|}
\hline Parameters & Group A & p-Value & Group B & p-Value & Group C & p-Value \\
\hline BMI & 0.090 & 0.636 & 0.383 & $0.051^{*}>>>-1$ & 0.387 & $0.05^{*}$ \\
\hline Age & -0.270 & 0.183 & -0.270 & 0.183 & -0.356 & 0.135 \\
\hline Waist circumference & 0.011 & 0.952 & 0.307 & 0.127 & 0.370 & $0.047^{\star}$ \\
\hline Hip circumference & 0.421 & $0.021^{\star}$ & 0.483 & $0.012^{*}$ & 0.521 & $0.022^{*}$ \\
\hline Waist-to-hip ratio & 0.028 & 0.876 & 0.206 & 0.221 & 0.472 & $0.031^{*}$ \\
\hline SBP & 0.391 & $0.033^{*}$ & 0.037 & 0.858 & 0.441 & $0.051^{*}$ \\
\hline DBP & 0.141 & 0.456 & 0.160 & 0.436 & 0.446 & $0.052^{\star}$ \\
\hline Fasting plasma glucose & 0.224 & 0.119 & 0.204 & 0.318 & 0.398 & 0.091 \\
\hline Two-hour plasma glucose & 0.101 & 0.546 & 0.421 & $0.021^{*}$ & 0.470 & $0.041^{*}$ \\
\hline HbA1c & 0.199 & 0.118 & -0.145 & 0.457 & -0.216 & 0.117 \\
\hline Cholesterol & -0.167 & 0.378 & 0.202 & 0.321 & 0.133 & 0.586 \\
\hline Triglyceride & 0.011 & 0.956 & -0.179 & 0.381 & 0.158 & 0.518 \\
\hline $\mathrm{HDL}$ & 0.146 & 0.441 & 0.146 & 0.457 & 0.286 & 0.235 \\
\hline LDL & 0.0 & 0.824 & 0.199 & 0.329 & .222 & 0.36 \\
\hline
\end{tabular}

\section{TABLE 2: Correlation of fasting glucagon with anthropometric and biochemical characteristics of}

Group A, Group B, and Group C

${ }^{*} \mathrm{p}$-Value $\leq 0.05$ is taken as significant.

BMI, body mass index; SBP, systolic blood pressure; DBP, diastolic blood pressure; HDL, high-density lipoprotein; LDL, low-density lipoprotein; HbA1c, glycated hemoglobin

Group A: Patients with normal glucose tolerance.

Group B: Patients with impaired glucose tolerance.

Group C: Patients with type 2 diabetes.

Multiple linear regression analysis derived 2-hPG, SBP, DBP, BMI, waist and hip circumference, and waist-tohip ratio as the independent predictors of fasting glucagon in Group C ( $\mathrm{p}<0.05)$. In Group B, 2-hPG, BMI, and hip circumference were evaluated as independent predictors of fasting glucagon, while SBP and hip circumference were found independently and positively associated with fasting glucagon in Group A ( $\mathrm{p}<$ 0.05) (Table 3). 


\section{Cureus}

\begin{tabular}{|c|c|c|c|}
\hline Studied groups & Clinical variables & Standardized coefficients $(\beta)$ & p-Value \\
\hline \multirow[t]{2}{*}{ Group A } & SBP & 0.391 & $0.033^{\star}$ \\
\hline & Hip circumference & 0.421 & $0.021^{*}$ \\
\hline \multirow[t]{3}{*}{ Group B } & Two-hour plasma glucose & 0.421 & $0.021^{\star}$ \\
\hline & BMI & 0.383 & $0.051^{\star}$ \\
\hline & Hip circumference & 0.483 & $0.012^{\star}$ \\
\hline \multirow[t]{7}{*}{ Group C } & Two-hour plasma glucose & 0.470 & $0.041^{\star}$ \\
\hline & SBP & 0.441 & $0.051^{\star}$ \\
\hline & DBP & 0.446 & $0.052^{\star}$ \\
\hline & BMI & 0.387 & $0.05^{*}$ \\
\hline & Waist circumference & 0.370 & $0.047^{\star}$ \\
\hline & Hip circumference & 0.521 & $0.022^{\star}$ \\
\hline & Waist-to-hip ratio & 0.472 & $0.031^{*}$ \\
\hline
\end{tabular}

\section{TABLE 3: Multiple linear regression analysis expressing independent predictors of fasting glucagon in Group A, Group B, and Group C}

${ }^{*} \mathrm{p}$-Value $\leq 0.05$ is taken as significant.

BMI, body mass index; SBP, systolic blood pressure; DBP, diastolic blood pressure

Group A: Patients with normal glucose tolerance.

Group B: Patients with impaired glucose tolerance.

Group C: Patients with type 2 diabetes.

\section{Discussion}

In the current study, we observed fasting glucagon level in patients with T2DM, impaired and normal glucose tolerance, and found insignificant differences in fasting glucagon level among study groups. This finding is consistent with the previous study indicating that glucose tolerance may not significantly affect fasting glucagon level [19]. However, conflicting finding has been reported, showing significantly higher fasting glucagon level in type 2 diabetic subjects [20]. A positive correlation of fasting glucagon with 2-hour postprandial plasma glucose levels in Groups B and C was found, thereby indicating dysregulated glucagon suppression after glucose intake. This finding is in line with the previous research that was done by AbdulGhani and DeFronzo [21]. Patients with T2DM have impaired postprandial glucagon suppression in the circumstances of defective insulin secretion and/or action [22]. However, it is of revelation in the present study that fasting glucagon was not correlated with FPG level and HbA1c level among any of the study groups. It is controversial as to whether there is a significant association between glucagon levels and glycemic control, as Knop et al. and Yoshiya et al. also validated our findings and suggested that fasting glucagon was not correlated with the FPG concentration in people with diabetes and impaired glucose tolerance, and, therefore, similar in diabetic and non-diabetic subjects [23,24]. Ortega et al. [25] reported that circulating glucagon levels were positively correlated with fasting glucose and $\mathrm{HbA1C}$ levels in obese subjects with impaired glucose tolerance. In contrast, Abdul-Ghani and DeFronzo found that glucagon concentration was inversely correlated with the FPG concentrations $(r=-0.35 ; P=0.001)$ during the OGTT, in T2DM participants [21]. This may be due to the difference in the measurement methods of glucagon levels or due to the specific characteristics of the study population.

Glucagon is known to increase energy expenditure through thermogenesis in brown adipose tissue [26]. Cegla et al. [27] reported that glucagon infusion increased the respiratory exchange ratio, resting energy expenditure rate, and carbohydrate and fat oxidation rates in healthy individuals. A systematic review and meta-analysis revealed a parallel decrease between fasting glucagon levels and weight loss in obese subjects [28]. In contrast, the current study identified a strong positive association of fasting glucagon level with BMI, waist circumference, waist-to-hip ratio in Group C, and hip circumference among all study groups. Thereby, it is seen that the appropriate balance between insulin and glucagon that is deficient in diabetes has a more 
favorable impact on weight control. It is also evident from the previous report that glucagon levels were elevated in high-hip circumference subjects and independently associated with other measures of abdominal obesity [20]. We also found the same outcomes and observed that glucagon was independently and positively associated with measures of central obesity in the current study. The role of glucagon in the pathophysiology of diabetes has long been recognized [8]. There are many modified molecules of glucagon that had the property to inhibit the function of glucagon receptors, thus preventing the hyperglucagonemiainduced hyperglycemia seen in diabetes [29]; however, the association of glucagon with abdominal obesity has been sparsely researched, and only a few studies are available. The present study indicated a positive association of fasting glucagon level with central obesity in type 2 diabetic subjects.

Research shows that glucagon acts primarily on the liver through regulation of hepatic lipid metabolism with reduction of the hepatic lipid accumulation and decrease in hepatic lipid secretion. Regarding the whole-body lipid metabolism, it is controversial to what extent glucagon influences lipolysis in adipose tissue, particularly in humans [30]. Physiologically, in the fasting state, exogenous glucagon reduces triglycerides, cholesterol levels, and release of LDL via the glucagon receptor with stimulated fatty acid oxidation, leading to the release of free fatty acids [30]. In contrast, Shajith et al. observed a significantly positive correlation of glucagon levels with LDL levels [20]. In the current study, we found no significant correlation of fasting glucagon level with lipid profile in the study population. The cause and significance of this observation are not clear; however, the altered liporegulatory role of glucagon due to excess adiposity in the study population may be contributory. The study is not without limitation. The role of glucagon in obese diabetic subjects would be more enlightening if a large sample size was selected for the research.

\section{Future recommendations}

Future studies with a large number of participants and other measurement parameters may further reinforce our current findings and help to understand the pathophysiological role of glucagon in diabetes. The sample size is small, especially for lean participants. The association of glucagon with clinical and anthropometric parameters would more enlighten with a large sample size and equal disparity of lean and obese subjects. The evaluation of other clinical parameters associated with glucose hemostasis might be assessed like insulin, insulin resistance, C-peptide, and glucagon-to-insulin ratio.

\section{Conclusions}

In summary, we report a positive association of glucagon level with 2-hour postprandial plasma glucose levels may indicate poor suppression of glucagon. Our findings also suggest that glucagon level is significantly associated with abdominal obesity in type 2 diabetic subjects; therefore, glucagon-suppressive drugs for the treatment of diabetes may have a significant effect on weight management. Additional studies are necessary to confirm and further clarify the clinical implications of fasting glucagon levels with glucose hemostasis and abdominal obesity.

\section{Additional Information}

\section{Disclosures}

Human subjects: Consent was obtained or waived by all participants in this study. Ethics Committee of Baqai Medical University issued approval BMU-EC/2016-04. This study was approved by the Ethics Committee of Baqai Medical University. Animal subjects: All authors have confirmed that this study did not involve animal subjects or tissue. Conflicts of interest: In compliance with the ICMJE uniform disclosure form, all authors declare the following: Payment/services info: All authors have declared that no financial support was received from any organization for the submitted work. Financial relationships: All authors have declared that they have no financial relationships at present or within the previous three years with any organizations that might have an interest in the submitted work. Other relationships: All authors have declared that there are no other relationships or activities that could appear to have influenced the submitted work.

\section{Acknowledgements}

We acknowledge the support of the statistician Miss Nida Mustafa from Baqai Institute of Diabetology and Endocrinology for data analysis.

\section{References}

1. Godoy-Matos AF: The role of glucagon on type 2 diabetes at a glance . Diabetol Metab Syndr. 2014, 6:91. 10.1186/1758-5996-6-91

2. Albrechtsen NJ, Hartmann B, Veedfald S, et al.: Hyperglucagonaemia analysed by glucagon sandwich ELISA: nonspecific interference or truly elevated levels?. Diabetologia. 2014, 57:1919-1926. 10.1007/s00125-0143283-z

3. Müller TD, Finan B, Clemmensen C, DiMarchi RD, Tschöp MH: The new biology and pharmacology of glucagon. Physiol Rev. 2017, 97:721-766. 10.1152/physrev.00025.2016

4. Ceriello A, Genovese S, Mannucci E, Gronda E: Glucagon and heart in type 2 diabetes: new perspectives . Cardiovasc Diabetol. 2016, 15:123. 10.1186/s12933-016-0440-3 
5. Henkel E, Menschikowski M, Koehler C, Leonhardt W, Hanefeld M: Impact of glucagon response on postprandial hyperglycemia in men with impaired glucose tolerance and type 2 diabetes mellitus. Metabolism. 2005, 54:1168-1173. 10.1016/j.metabol.2005.03.024

6. Ahrén B: Beta- and alpha-cell dysfunction in subjects developing impaired glucose tolerance: outcome of a 12-year prospective study in postmenopausal Caucasian women. Diabetes. 2009, 58:726-731. 10.2337/db081158

7. Kahn SE, Hull RL, Utzschneider KM: Mechanisms linking obesity to insulin resistance and type 2 diabetes . Nature. 2006, 444:840-846. 10.1038/nature05482

8. Kulina GR, Rayfield EJ: The role of glucagon in the pathophysiology and management of diabetes . Endocr Pract. 2016, 22:612-621. 10.4158/EP15984.RA

9. Stern JH, Smith GI, Chen S, Unger RH, Klein S, Scherer PE: Obesity dysregulates fasting-induced changes in glucagon secretion. J Endocrinol. 2019, 243:149-160. 10.1530/JOE-19-0201

10. Tan TM, Field BC, McCullough KA, et al.: Coadministration of glucagon-like peptide-1 during glucagon infusion in humans results in increased energy expenditure and amelioration of hyperglycemia. Diabetes. 2013, 62:1131-1138. 10.2337/db12-0797

11. Stahel P, Lee SJ, Sud SK, Floh A, Dash S: Intranasal glucagon acutely increases energy expenditure without inducing hyperglycaemia in overweight/obese adults. Diabetes Obes Metab. 2019, 21:1357-1364. 10.1111/dom.13661

12. American Diabetes Association. Accessed: November 1, 2020: http://www.diabetes.org/a1c/diagnosis.

13. Ho-Pham LT, Lai TQ, Nguyen MTT, Nguyen TV: Relationship between body mass index and percent body fat in Vietnamese: implications for the diagnosis of obesity. PLoS One. 2015, 10:e0127198. 10.1371/journal.pone.0127198

14. Jaeschke L, Steinbrecher A, Pischon T: Measurement of waist and hip circumference with a body surface scanner: feasibility, validity, reliability, and correlations with markers of the metabolic syndrome. PLoS One. 2015, 10:e0119430. 10.1371/journal.pone.0119430

15. Basit A, Alvi SFD, Fawwad A, Ahmed K, Ahmedani MY, Hakeem R: Temporal changes in the prevalence of diabetes, impaired fasting glucose and its associated risk factors in the rural area of Baluchistan. Diabetes Res Clin Pract. 2011, 94:456-462. 10.1016/j.diabres.2011.08.009

16. Sharma SP, Anjankar AP, Kale A: Comparison of glucose levels using glucometer and GOD-POD method in diabetic patients. Int J Clin Biochem Res. 2017, 4:6-10. 10.18231/2394-6377.2017.0002

17. Fawwad A, Sabir R, Riaz M, Moin H, Basit A: Measured versus calculated LDL-cholesterol in subjects with type 2 diabetes. Pak J Med Sci. 2016, 32:955-960. 10.12669/pjms.324.9896

18. Pundir CS, Chawla S: Determination of glycated hemoglobin with special emphasis on biosensing methods . Anal Biochem. 2014, 444:47-56. 10.1016/j.ab.2013.09.023

19. Hsieh J, Longuet C, Baker CL, Qin B, Federico LM, Drucker DJ, Adeli K: The glucagon-like peptide 1 receptor is essential for postprandial lipoprotein synthesis and secretion in hamsters and mice. Diabetologia. 2010, 53:552-561. 10.1007/s00125-009-1611-5

20. Anoop S, Misra A, Bhatt SP, Gulati S, Mahajan H, Prabakaran G: High plasma glucagon levels correlate with waist-to-hip ratio, suprailiac skinfold thickness, and deep subcutaneous abdominal and intraperitoneal adipose tissue depots in nonobese Asian Indian males with type 2 diabetes in North India. J Diabetes Res. 2017, 2017:2376016. 10.1155/2017/2376016

21. Abdul-Ghani M, DeFronzo RA: Fasting hyperglycemia impairs glucose- but not insulin-mediated suppression of glucagon secretion. J Clin Endocrinol Metab. 2007, 92:1778-1784. 10.1210/jc.2006-1515

22. Liu Z, Kim W, Chen Z, et al.: Insulin and glucagon regulate pancreatic $\alpha$-cell proliferation. PLoS One. 2011, 6:e16096. 10.1371/journal.pone.0016096

23. Hosokawa Y, Kozawa J, Nishizawa H, et al.: Positive correlation between fasting plasma glucagon and serum C-peptide in Japanese patients with diabetes. Heliyon. 2019, 5:e01715. 10.1016/j.heliyon.2019.e01715

24. Knop FK, Aaboe K, Vilsbøll T, Vølund A, Holst JJ, Krarup T, Madsbad S: Impaired incretin effect and fasting hyperglucagonaemia characterizing type 2 diabetic subjects are early signs of dysmetabolism in obesity. Diabetes Obes Metab. 2012, 14:500-510. 10.1111/j.1463-1326.2011.01549.x

25. Ortega FJ, Moreno-Navarrete JM, Sabater M, Ricart W, Frühbeck G, Fernández-Real JM: Circulating glucagon is associated with inflammatory mediators in metabolically compromised subjects. Eur J Endocrinol. 2011, 165:639-645. 10.1530/EJE-11-0384

26. Campbell JE, Drucker DJ: Islet $\alpha$ cells and glucagon--critical regulators of energy homeostasis . Nat Rev Endocrinol. 2015, 11:329-338. 10.1038/nrendo.2015.51

27. Cegla J, Troke RC, Jones B, et al.: Coinfusion of low-dose GLP-1 and glucagon in man results in a reduction in food intake. Diabetes. 2014, 63:3711-3720. 10.2337/db14-0242

28. Silvestre MP, Goode JP, Vlaskovsky P, McMahon C, Tay A, Poppitt SD: The role of glucagon in weight lossmediated metabolic improvement: a systematic review and meta-analysis. Obes Rev. 2018, 19:233-253. 10.1111/obr.12631

29. Lotfy M, Kalasz H, Szalai G, Singh J, Adeghate E: Recent progress in the use of glucagon and glucagon receptor antagonists in the treatment of diabetes mellitus. Open Med Chem J. 2014, 8:28-35. 10.2174/1874104501408010028

30. Galsgaard KD, Pedersen J, Knop FK, Holst JJ, Wewer Albrechtsen NJ: Glucagon receptor signaling and lipid metabolism. Front Physiol. 2019, 10:413. 10.3389/fphys.2019.00413 